\title{
Hábitos de sono e relação com indicadores de saúde mental na infância
}

\author{
Thaísa Gios, Tatiana P. Mecca, Juliana Akemi, Lucas E. Kataoka e Rosane Lowenthal
} Departamento de Saúde Mental, Faculdade de Ciências Médicas da Santa Casa de São Paulo (FCMSCSP)

Submissão: 28 abr. 2020.

Aceite: 9 ago. 2021.

$$
\begin{aligned}
& \text { Nota dos autores } \\
& \text { Thaísa Gios (D) https://orcid.org/o0oo-0002-9408-3403 } \\
& \text { Tatiana P. Mecca (D) https://orcid.org/0000-0002-2009-6228 } \\
& \text { Juliana Akemi (D) https://orcid.org/0000-0003-4038-833X } \\
& \text { Lucas E. Kataoka (D) https://orcid.org/00oo-0001-7117-3919 } \\
& \text { Rosane Lowenthal (D) https://orcid.org/00oo-0002-6330-850X }
\end{aligned}
$$

Financiamento: Programa Institucional de Bolsa de Iniciação Científica (Pibic) e de mestrado FCMSCSP.

Correspondências referentes a este artigo devem ser enviadas para Rosane Lowenthal, Departamento de Saúde Mental, Faculdade de Ciências Médicas da Santa Casa de São Paulo. Rua Dona Veridiana, 55, $3^{\circ}$ andar, São Paulo, SP, Brasil. CEP 01238-010. Brasil. E-mail: rosane.lowenthal@gmail.com 


\title{
Resumo
}

Este estudo investigou a relação entre hábitos de sono e indicadores de saúde mental na infância relatados por cuidadores, além de buscar evidências de validade e precisão da adaptação para o Brasil do Children's Sleep Habits Questionnaire (CSHQ). Participaram 60 crianças, entre 4 e 10 anos, de uma escola pública da região central de São Paulo, em 2019. A média geral no CSHQ-BR foi 49,08. Não houve diferenças de sexo nos escores do CSHQ e do Strengths and Difficulties Questionnaire (SDQ). Houve correlações positivas e significativas entre o $\mathrm{CSHQ}$ e o SDQ, especificamente entre dificuldades, parassonias e distúrbios respiratórios do sono com problemas emocionais e hiperatividade. Hábitos de sono explicaram 23\% da variância no SDQ. O coeficiente alfa de Cronbach do CSHQ-BR foi 0,75, indicando consistência interna adequada. Esses achados apontam evidências de validade e precisão do CSHQ-BR. Hábitos de sono estão associados a indicadores de problemas emocionais e comportamentais.

Palavras-chave: sono, saúde mental, infância, problemas internalizantes, problemas externalizantes

\section{SLEEP HABITS AND RELATIONSHIP WITH MENTAL HEALTH INDICATORS IN CHILDHOOD}

\begin{abstract}
The relationship was investigated between sleep habits and mental health indicators in childhood reported by caregivers, in addition to seeking evidence of validity and reliability of the Children's Sleep Habits Questionnaire (CSHQ), Brazilian version. 60 children participated, between 4 and 10 years old, from a public school in the central region of São Paulo, in 2019. The overall mean of the CSHQ-BR total score was 49.08. There were no differences between sex in the CSHQ and Strengths and Difficulties Questionnaire (SDQ) scores. Positive and significant correlations were observed between the CSHQ and SDQ, specifically between difficulties, parasomnias, sleep-disordered breathing with emotional problems and hyperactivity. Sleep problems explain $23 \%$ of the variance of the SDQ scores. The Cronbach's alpha coefficient was 0.75 , indicating adequate internal consistency. These findings point to evidence of the validity and accuracy of the CSHQ-BR. Sleep habits are associated with indicators of emotional and behavioral problems.
\end{abstract}

Keywords: sleep, mental health, childhood, internalizing problems, externalizing problems

\section{HÁBITOS DE SUEÑO Y RELACIÓN CON INDICADORES DE SALUD MENTAL EN LA INFANCIA}

\section{Resumen}

Se investigó la relación entre hábitos de sueño y indicadores de salud mental infantil reportados por cuidadores, además de buscar evidencia de validez y precisión de la adaptación del Cuestionario de Hábitos de Sueño Infantil (CSHQ) para Brasil. En 2019 participaron 60 niños de 4 a 10 años de una escuela pública de la región central de São Paulo. El promedio general en el CSHQ-BR fue 49,08. No hubo diferencias de género en las puntuaciones del CSHQ y del Cuestionario de Fortalezas y Dificultades 
(SDQ). Hubo correlaciones positivas y significativas entre CSHQ y SDQ, específicamente entre dificultades, parasomnias y trastornos respiratorios del sueño con problemas emocionales e hiperactividad. Hábitos de sueño explicaron $23 \%$ de la variación en el SDQ. El coeficiente alfa de Cronbach de CSHQ-BR fue 0,75 , indicando una consistencia interna adecuada. Estos hallazgos apuntan evidencia de validez y precisión del CSHQ-BR. Hábitos de sueño están asociados con indicadores de problemas emocionales y de comportamiento.

Palabras clave: sueño, salud mental, infancia, problemas de internalización, problemas de externalización 
O sono é um fenômeno complexo e fundamental para o desenvolvimento saudável. Interfere no comportamento, na consolidação da memória e de outros aspectos cognitivos, na regulação metabólica, hormonal e emocional. Quando inadequado, é fator de risco para obesidade, diabetes, doenças cardíacas, distúrbios na imunidade e transtornos de humor, interferindo no bem-estar físico e mental do indivíduo, acarretando prejuízo funcional e nas relações interpessoais (Barbisan \& Bueno, 2019; Neves et al., 2017; Silva et al., 2014).

As dificuldades de sono são comuns na infância, atingindo cerca de 20\%-30\% das crianças com desenvolvimento típico e $80 \%$ das crianças com alterações do neurodesenvolvimento (Damiani et al., 2014; Moore et al., 2017). Quadros de ansiedade e depressão, transtorno de déficit de atenção/hiperatividade (TDAH) e transtorno do espectro autista (TEA) ocorrem com frequência em associação ou como comorbidade ao quadro de insônia (Nunes \& Bruni, 2015; Vaughn et al., 2015; Liu et al., 2014). Estima-se, por exemplo, que $25 \%$ a $50 \%$ das crianças com TDAH apresentem transtornos do sono (Miano et al., 2012; Paavonen et al., 2009).

Estudos de diferentes países mostram que a associação entre transtornos do sono e problemas emocionais/comportamentais em crianças parece ser universal (Nunes \& Bruni, 2015; Wu et al., 2016; Wang et al., 2020; Whalen et al., 2016). Aspectos relacionados à curta duração do sono ou à irregularidade na hora de dormir estão associados a problemas de atenção, comportamentos agressivos, hiperatividade e sintomas emocionais (Wu et al., 2016; Wang et al., 2020; Whalen et al., 2016; Schlarb et al., 2016). Ademais, crianças com transtornos do sono apresentam maiores alterações não adaptativas no processo de geração e regulação de emoções, mais dificuldades de relacionamento entre pares e pior prontidão para a aprendizagem com impacto no rendimento escolar (Tso et al., 2016).

Pesquisas indicam que transtornos do sono em crianças são preditores significativos de problemas emocionais e comportamentais posteriores, como ansiedade, depressão, queixas somáticas, problemas de atenção, funções executivas menos desenvolvidas e comportamentos agressivos (Nelson et al., 2018; Whalen et al., 2016; Nunes \& Bruni, 2015; Owens \& Mindell, 2011).

As apresentações clínicas dos transtornos de sono são variáveis. Durante os primeiros anos, são frequentes as queixas de dificuldades para iniciar o sono e/ou despertares noturnos. Após esse período observam-se parassonias (tais como despertar confusional, sonambulismo e terror noturno) e distúrbios respiratórios do sono (como apneia obstrutiva do sono). A partir da idade pré-escolar, ocorrem os distúrbios relacionados a questões de higiene do sono inadequada, e, na adolescência, há os transtornos relacionados a questões circadianas ou a movimentos excessivos durante o sono (Nunes \& Bruni, 2015).

Um estudo recente que investigou problemas do sono e de saúde mental em crianças pré-escolares na China e no Japão mostrou associação entre problemas emocionais e compor- 
tamentais com transtornos de sono. Entretanto, houve diferença nos aspectos de saúde mental e do sono entre os países. Por exemplo, crianças chinesas com dificuldades do sono apresentam mais problemas de relacionamento com pares. Na China, os problemas do sono mais frequentes são respiratórios e sonolência diurna, enquanto no Japão são ansiedade do sono e despertar noturno (Wang et al., 2020). Isso indica que diferenças culturais podem impactar de formas distintas a relação entre características específicas de sono e indicadores de saúde mental na infância.

A detecção precoce dos transtornos do sono e fatores de risco para transtornos psiquiátricos é fundamental, pois propicia o manejo adequado e consequentemente o prognóstico favorável, evitando a cronicidade das doenças. Instrumentos para rastreio, como o Children's Sleep Habits Questionnaire (CSHQ) e o Questionário de Capacidades e Dificuldades (Strengths and Difficulties Questionnaire - SDQ), podem auxiliar na prática diária, já que são de fácil e rápida aplicação.

O CSHQ é um questionário sobre hábitos de sono desenvolvido por Owens et al. (2000), amplamente utilizado nos estudos internacionais sobre sono na infância. Os itens e a estrutura do questionário são baseados em apresentações clínicas comuns dos diagnósticos mais prevalentes segundo a International Classification of Sleep Disorders e avaliam a percepção dos pais sobre o sono dos filhos.

Existem adaptações desse questionário para diversos idiomas, como chinês (Liu, Liu, \& Wang, 2003), hebraico (Tzchishinsky et al., 2008), holandês (Wauman et al., 2010), alemão (Schlarb et al., 2010), espanhol (Cruz et al., 2016) e português de Portugal (Silva et al., 2014), e, para a maior parte deles, existem estudos prévios de evidências de validade. No Brasil, a tradução, a adaptação transcultural e os primeiros estudos psicométricos foram realizados recentemente (Gios, 2020).

Em relação ao rastreio de problemas de saúde mental na infância, há o SDQ composto por um total de 25 itens divididos em cinco subescalas. A versão brasileira do SDQ apresenta estudos nacionais prévios de evidência de validade para uso em triagem de saúde mental na infância (Saur \& Loureiro, 2012; Silva et al., 2015). Os escores do SDQ permitem identificar sintomas internalizantes e problemas externalizantes de comportamento (Goodman, 1997).

Por conta da associação entre distúrbios do sono e problemas comportamentais em crianças, e em razão da escassez de estudos nacionais que utilizem instrumentos de rastreio consolidados pela literatura, o objetivo deste trabalho é verificar a relação dos padrões do sono com indicadores de saúde mental na infância relatados por cuidadores. Acrescenta-se ainda que os dados oriundos do presente estudo proverão evidências de validade baseadas na relação com variáveis externas da adaptação para o português do Brasil do CSHQ. De acordo com a revisão feita da literatura, a hipótese é de que sejam observadas correlações de magnitude 
baixa a moderada entre problemas de sono e aumento de problemas de saúde mental em crianças conforme relato dos cuidadores.

\section{Método}

\section{Desenho e aspectos gerais do estudo}

Trata-se de um estudo transversal, observacional, descritivo e correlacional. A coleta de dados foi realizada em 2019, durante uma reunião de pais em uma escola pública da região central de São Paulo. Durante o encontro, os cuidadores foram convidados a assistir a palestras sobre sono e preencher os questionários, com auxílio de uma equipe composta por estudantes de Medicina e Fonoaudiologia, previamente capacitada para aplicação dos instrumentos.

\section{Participantes}

A amostra foi selecionada por conveniência. Participaram deste estudo 60 crianças (53,3\% meninos), com idades entre 4 e 10 anos e 11 meses $(M=7,66$ anos e $D P=1,36)$, todas estudantes de uma escola pública da cidade de São Paulo. Conforme o critério de Classificação Econômica Brasil da Associação Brasileira de Empresas de Pesquisa - Abep (2019), a média da população estudada encontra-se no estrato socioeconômico $C_{1}(M=25,78)$, com renda média domiciliar estimada em 3.085, 48 reais. Do total, 70\% dos questionários foram preenchidos pelas mães, $13,3 \%$ pelos pais, $10 \%$ pelos avós, e $6,7 \%$ não responderam a esse quesito.

\section{Critérios de inclusão e exclusão}

O critério de inclusão dos cuidadores (pais e/ou responsáveis) foi permanecer com criança, no mínimo, seis noites por semana. Excluíram-se do estudo crianças cujos cuidadores referiram síndromes genéticas, transtornos psiquiátricos ou uso contínuo de psicofármacos.

\section{Instrumentos}

- CSHQ: neste estudo, utilizou-se a versão já traduzida e em processo de evidências de validade para o português do Brasil do CSHQ - versão abreviada (Gios, 2020). Esse questionário inclui itens relacionados a domínios-chave que abrangem as principais queixas clínicas de sono na faixa etária pediátrica. Os 33 itens são agrupados conceitualmente em oito subescalas, refletindo os seguintes domínios do sono: resistência em ir para a cama, início do sono, duração do sono, ansiedade do sono, despertares noturnos, parassonias, distúrbios respiratórios do sono e sonolência diurna. É utilizado para a triagem dos distúrbios do sono em crianças entre 4 e 10 anos de idade. Esse limite de idade foi definido com o objetivo de minimizar os possíveis efeitos das mudanças puberais no comportamento do sono. A frequência dos comportamentos do sono é classificada em uma escala de 
três pontos, como "habitualmente" (ocorre de cinco a sete vezes por semana), "às vezes" (ocorre de duas a quatro vezes por semana) ou "raramente" (de zero a uma vez por semana) (Owens et al., 2000). O CSHQ-BR encontra-se descrito no Apêndice 1.

- Questionário de Capacidades e Dificuldades (SDQ): instrumento utilizado para rastreio de problemas de saúde mental na infância. É constituído por 25 itens divididos em cinco subescalas: problemas no comportamento pró-social, hiperatividade, problemas emocionais, problemas de conduta e de relacionamento, com cinco itens em cada subescala. As respostas podem ser: falso (zero ponto), mais ou menos verdadeiro (um ponto) ou verdadeiro (dois pontos), e cada item recebe uma pontuação específica. A exceção ocorre na subescala de comportamento pró-social, na qual quanto maior a pontuação, menor é a quantidade de queixas. Para cada uma das cinco subescalas do SDQ, a pontuação pode variar de o a 10, sendo a pontuação do escore total de dificuldades gerada pela soma dos resultados de todas as subescalas, exceto a de sociabilidade, variando de 0 a 40 pontos (Goodman, 1997).

- Critério de Classificação Econômica Brasil da Abep (2019): apresenta uma classificação de nível socioeconômico, considerando todos os bens que estão dentro do domicílio (independentemente da forma de aquisição), o grau de instrução e a condições de moradia. Possui uma estratificação da população em sete níveis socioeconômicos (NSE).

\section{Procedimentos e considerações éticas}

O estudo foi aprovado pelo Comitê de Ética da Irmandade da Santa Casa de Misericórdia de São Paulo. A avaliação dos problemas de sono e o rastreio de saúde mental foram aprovados em dois projetos individuais: Certificado de Apresentação para Apreciação Ética (CAAE no 99698918.1.0000.5479 e CAAE no 20689919.3.0000.5479). O responsável pela instituição onde ocorreu a coleta de dados e os responsáveis pelas crianças assinaram o Termo de Consentimento Livre e Esclarecido (TCLE), conforme preconiza a Resolução nº 510/2016 do Conselho Nacional de Saúde para pesquisas com seres humanos. Os participantes foram informados sobre os procedimentos e a confidencialidade dos dados coletados, e também sobre a divulgação anônima dos resultados.

\section{Análise de dados}

Os dados foram analisados por meio do software Statistical Package for Social Sciences (SPSS) - versão 21.0. Realizaram-se estatísticas descritivas (média e desvio padrão) com a finalidade de caracterizar a amostra quanto aos escores nos questionários. Para verificar a 
adequação da distribuição dos dados e definir o tipo de análise inferencial a ser conduzida, realizou-se o teste Kolmogorov-Smirnov e descreveram-se os valores de assimetria e curtose dos escores do SDQ e CSHQ. Os valores de assimetria para o SDQ variaram de -1 a 1, com exceção do comportamento pró-social, enquanto os valores de curtose se distanciaram do esperado. Já no CSHQ, parte dos valores das subescalas está assimétrica e encontra-se distante do esperado. Por fim, o teste Kolmogorov-Smirnov apresentou significância estatística ( $p \leq 0$ ,05), e, por isso, optou-se pelo uso de análises não paramétricas.

Foram comparados os escores de meninos e meninas no SDQ e no CSHQ pelo teste de Mann-Whitney. Realizaram-se a análise de correlação de Spearman entre os escores no SDQ e CSHQ, e, por fim, a análise de regressão linear, considerando distúrbios do sono como variável independente e problemas de comportamento como desfecho. A precisão do instrumento para essa amostra foi obtida por meio da análise de consistência interna, especificamente pelo coeficiente alfa de Cronbach.

\section{Resultados}

Inicialmente foram realizadas análises descritivas por sexo, bem como para amostra total dos escores do SDQ e CSHQ. Os resultados encontram-se descritos na Tabela 1.

\section{Tabela 1}

Estatísticas descritivas dos escores do SDQ e CSHQ

\begin{tabular}{|c|c|c|c|c|c|c|}
\hline Subescalas & $\begin{array}{c}\text { Meninas } \\
\text { M(DP) }\end{array}$ & $\begin{array}{c}\text { Meninos } \\
\text { M(DP) }\end{array}$ & $\begin{array}{c}\text { Geral } \\
\text { M(DP) }\end{array}$ & Assimetria & Curtose & $\begin{array}{l}\text { Mín.- } \\
\text {-máx. }\end{array}$ \\
\hline S. Emocionais & $4,56(2,60)$ & $3,92(2,81)$ & $4,28(2,69)$ & 0,23 & $-0,89$ & $0-10$ \\
\hline P. Conduta & $1,97(1,86)$ & $2,64(1,89)$ & $2,29(1,89)$ & 0,54 & $-0,57$ & $0-7$ \\
\hline Hiperatividade & $4,12(2,67)$ & $4,78(2,51)$ & $4,43(2,59)$ & 0,31 & $-0,73$ & $0-10$ \\
\hline $\begin{array}{l}\text { Relaciona- } \\
\text { mento }\end{array}$ & $1,87(1,77)$ & $1,67(1,76)$ & $1,78(1,75)$ & 0,90 & $-0,03$ & $0-6$ \\
\hline Pró-social & $9,10(1,42)$ & $9,18(1,24)$ & $9,14(1,33)$ & $-2,74$ & 9,89 & $3-10$ \\
\hline Total SDQ & $12,40(6,04)$ & $12,75(5,73)$ & $12,56(5,85)$ & 0,44 & $-0,16$ & $1-27$ \\
\hline Resistência & $9,72(2,80)$ & $9,59(2,51)$ & $9,70(2,63)$ & 0,71 & $-0,07$ & $6-16$ \\
\hline Início do Sono & $1,50(0,74)$ & $1,38(0,71)$ & $1,43(0,72)$ & 1,36 & 0,34 & $1-3$ \\
\hline Duração & $4,26(1,57)$ & $4,10(1,57)$ & $4,18(1,54)$ & 1,09 & $-0,11$ & $3-8$ \\
\hline Ansiedade & $6,75(2,34)$ & $6,14(2,39)$ & $6,48(2,35)$ & 0,69 & $-0,61$ & $4^{-12}$ \\
\hline D. Noturno & $4,14(1,29)$ & $3,76(0,86)$ & $3,98(1,11)$ & 1,37 & 2,34 & $3-8$ \\
\hline
\end{tabular}




\begin{tabular}{|c|c|c|c|c|c|c|}
\hline Subescalas & $\begin{array}{l}\text { Meninas } \\
\text { M(DP) }\end{array}$ & $\begin{array}{l}\text { Meninos } \\
\text { M(DP) }\end{array}$ & $\begin{array}{l}\text { Geral } \\
M(D P)\end{array}$ & Assimetria & Curtose & $\begin{array}{l}\text { Mín.- } \\
\text {-máx. }\end{array}$ \\
\hline Parassonias & $10,48(2,29)$ & $10,42(2,55)$ & $10,44(2,38)$ & 0,31 & $-0,64$ & $7-16$ \\
\hline Distúrbios Res. & $4,24(1,37)$ & $4,39(1,85)$ & $4,32(1,60)$ & 1,20 & 0,63 & $3-9$ \\
\hline Sonolência D & $14,75(3,38)$ & $13,22(3,76)$ & $14,07(3,61)$ & 0,27 & $-0,71$ & $8-21$ \\
\hline Total CSHQ & $48,90(13,57)$ & $49,00(9,86)$ & $49,08(11,80)$ & $-1,39$ & 4,04 & $2-68$ \\
\hline
\end{tabular}

Nota. S. Emocionais - sintomas emocionais; P. Conduta - problemas de conduta; Relacionamento - problemas de relacionamento com colegas; Pró-social - comportamentos pró-sociais; Resistência - resistência em ir para a cama; Duração - duração do sono; Ansiedade. ansiedade do sono; D. Noturno - despertar noturno; Distúrbios Res. - distúrbios respiratórios; Sonolência $\mathrm{D}$ - sonolência diurna.

Em seguida, o teste de Mann-Whitney foi conduzido com a finalidade de comparar os escores de meninos e meninas. Os resultados indicaram não haver diferença estatisticamente significativa entre ambos os grupos para sintomas emocionais $[(U)=357,5 ; p=0,35]$, problemas de conduta $[(U)=328 ; p=0,14]$, hiperatividade $[(U)=367 ; p=0,22]$, dificuldades de relacionamento $[(U)=416 ; p=0,63]$ e comportamento pró-social $[(U)=428,5 ; p=0,92]$. Do mesmo modo, não foram observadas diferenças estatisticamente significativas entre meninos e meninas para os índices de sono, entre eles: resistência em ir para a cama $[(U)=385$; $p=0,92]$, início do sono $[(U)=395 ; p=0,46]$, duração do sono $[(U)=397,5 ; p=0,70]$, ansiedade do sono $[(U)=328,5 ; p=0,20]$, despertar noturno $[(U)=314,5 ; p=0,36]$, parassonias $[(U)=388,5 ; p=0,77]$, distúrbios respiratórios do sono $[(U)=391 ; p=0,80]$ e sonolência diurna $[(U)=294 ; p=0,10]$. Não houve, portanto, diferenças de escores no CSHQ e no SDQ que justificassem o controle dessa variável em outras análises.

Em seguida, foram conduzidas correlações de Spearman entre os escores do CSHQ com os escores do SDQ respondido pelos pais, com a idade das crianças (em meses) e com a pontuação total na Abep como indicador de NSE. Os resultados das análises de correlação encontram-se descritos na Tabela 2.

Com relação ao nível socioeconômico, houve correlação negativa, significativa e de baixa magnitude entre resistência em ir para a cama e pontuação total do questionário Abep, indicando que quanto menor o nível socioeconômico, maior a resistência em ir para a cama. Já em relação à idade, encontrou-se correlação negativa, significativa e de magnitude moderada entre horas de sono e idade em meses, indicando que quanto menor a idade da criança, mais horas de sono, enquanto o aumento da idade se relaciona com diminuição da quantidade de horas de sono. Observou-se também correlação positiva, significativa e de magnitude baixa entre início de sono e idade, ou seja, quanto maior a idade, o início do sono é mais tardio. 
Ainda em relação à idade, foi observada uma tendência para correlação positiva com sonolência diurna.

\section{Tabela 2}

Correlação de Spearman entre os escores no CSHQ com as variáveis, idade, escores no SDQ e Abep

\begin{tabular}{|c|c|c|c|c|c|c|c|c|}
\hline Sono & Idade & S. E & P. C & Hip. & Rel. C. & Pró-social & SDQ & Abep \\
\hline Hora de dormir & $-0,04$ & 0,01 & $-0,17$ & 0,15 & 0,24 & $-0,01$ & 0,12 & 0,11 \\
\hline Horas de sono & $-0,40 * *$ & $-0,10$ & $-0,19$ & 0,00 & $-0,14$ & 0,04 & $-0,10$ & 0,10 \\
\hline Resistência & $-0,07$ & 0,23 & 0,07 & $-0,03$ & $-0,09$ & $-0,08$ & 0,09 & $-0,30 *$ \\
\hline Início do sono & $0,27 *$ & 0,01 & 0,16 & $0,28 *$ & 0,11 & $-0,21$ & 0,23 & $-0,04$ \\
\hline Duração do sono & 0,01 & 0,25 & 0,18 & 0,19 & 0,17 & $-0,10$ & $0,31 *$ & $-0,09$ \\
\hline $\begin{array}{l}\text { Ansiedade } \\
\text { do sono }\end{array}$ & $-0,13$ & 0,21 & $-0,06$ & $-0,02$ & $-0,15$ & 0,10 & 0,05 & $-0,10$ \\
\hline $\begin{array}{l}\text { Despertar } \\
\text { noturno }\end{array}$ & 0,23 & 0,11 & 0,00 & $-0,13$ & $-0,18$ & 0,08 & $-0,02$ & $-0,03$ \\
\hline Parassonia & $-0,13$ & $0,51 * *$ & 0,20 & $0,37 * *$ & 0,24 & $-0,21$ & $0,51 * *$ & $-0,15$ \\
\hline $\begin{array}{l}\text { Distúrbio } \\
\text { respiratório }\end{array}$ & $-0,06$ & $0,41 * *$ & 0,04 & $0,38 * *$ & 0,18 & $-0,21$ & $0,44^{* *}$ & 0,00 \\
\hline $\begin{array}{l}\text { Sonolência } \\
\text { diurna }\end{array}$ & 0,24 & 0,15 & 0,02 & 0,17 & 0,14 & $-0,13$ & 0,22 & 0,10 \\
\hline CSHQ & 0,08 & $0,41 * *$ & 0,15 & 0,25 & 0,20 & $-0,09$ & $0,45^{* *}$ & $-0,06$ \\
\hline
\end{tabular}

Nota. S. E - sintomas emocionais; P. C - problemas de conduta; Hip - desatenção e hiperatividade; Rel. C - problemas de relacionamento com colegas; Pró-social - comportamentos pró-sociais; SDQ - escore total de problemas de comportamento no SDQ; Abep - classificação socioeconômica.

* Correlação é significativa ao nível de $p \leq 0,05$.

** Correlação é significativa ao nível de $p \leq 0,01$.

Itálico: tendência $(0,05<p \leq 0,07)$.

Observou-se correlação positiva, significativa e magnitude moderada entre o escore total no CSHQ e o escore total no SDQ. Quando se analisaram os domínios específicos do $\mathrm{CSHQ}$, notou-se que a duração sono e a presença de parassonias e distúrbios respiratórios se correlacionaram de forma positiva, significativa e com magnitude moderada com os escores totais no SDQ.

No que tange aos domínios específicos do SDQ, não foram observadas correlações significativas entre os diferentes aspectos do sono com problemas de conduta, relacionamento com colegas e comportamento pró-social. No caso de problemas de relacionamento com colegas, houve uma tendência de baixa correlação com parassonias. Contudo, correlação po- 
sitiva, significativa e de baixa magnitude foi observada entre sintomas de desatenção/hiperatividade com o início do sono, parassonias e distúrbios respiratórios do sono. Nota-se que o maior tempo para início do sono e presença de parassonias ou distúrbios respiratórios relacionam-se com maior frequência de sintomas de desatenção/hiperatividade. Esses escores também apresentaram tendência de baixa correlação com os escores totais no CSHQ.

Encontrou-se correlação positiva, significativa e de magnitude moderada entre sintomas emocionais com parassonias e distúrbios respiratórios do sono. Dessa forma, observa-se que quanto mais sintomas de parassonias e alterações respiratórias do sono a criança apresentar, mais dificuldades emocionais são relatadas pelos cuidadores.

A análise de regressão possibilitou a identificação de um modelo que apresentou um coeficiente de determinação de 0,232 . Dessa forma, verificou-se que as características do sono medidas pelo CSHQ explicam $23 \%$ da variância dos escores observados em problemas comportamentais que são indicadores de saúde em crianças entre 4 e 10 anos de idade. A Tabela 3 apresenta o modelo resultante da regressão.

\section{Tabela 3}

Sumário do modelo oriundo da regressão

\begin{tabular}{ccccccc}
\hline & & \multicolumn{5}{c}{ Estimativa de } \\
Modelo & $\mathbf{R}$ & $\mathbf{R}^{2}$ & $\mathbf{R}^{2}$ ajustado & erro padrão & $\mathbf{F}$ & $\boldsymbol{p}$ \\
\hline 1 & 0,482 & 0,232 & 0,219 & 5,22 & 17,263 & $<0,001$ \\
\hline
\end{tabular}

Por fim, o presente estudo é o primeiro conduzido com a versão brasileira do CSHQ-BR, que foi previamente traduzida e adaptada por Gios (2020). Estudos prévios com a versão portuguesa, o CSHQ-PT (Silva et al., 2014), também foram conduzidos no Brasil (Loekmanwidjaja et al., 2018; Urrutia-Pereira et al., 2017). Todavia, neste estudo optou-se por utilizar a versão com adaptação transcultural para o Brasil, tendo em vista a necessidade do uso de instrumentos devidamente adaptados para o contexto no qual são utilizados (Borsa et al., 2012). Realizou-se análise de consistência interna do instrumento para a presente amostra, a qual indicou um coeficiente alfa de Cronbach de 0,75. Esse valor é considerado adequado, indicando boa correlação item-total dos itens que compõem o instrumento.

\section{Discussão}

Tendo em vista estudos prévios em diferentes populações no mundo que relatam a associação entre sono e saúde mental na infância (Wu et al., 2016; Liu et al., 2014; Wang et al., 2020) e a escassez de questionários disponíveis em contexto nacional para avaliação de características de sono, o presente estudo objetivou investigar a relação entre características que são indicadoras de problemas de sono com uma medida de rastreio de sintomas internalizan- 
tes e externalizantes utilizada como triagem para saúde mental em crianças. Acrescenta-se ainda o fato de serem utilizadas ferramentas de rastreio amplamente empregadas em diversos estudos nacionais e internacionais, tendo como contribuição os primeiros achados de evidências de validade e precisão da versão brasileira do CSHQ.

De acordo com classificação previamente estabelecida para os escores do SDQ (Goodman, 1997), em média as crianças apresentaram-se como limítrofes para sintomas emocionais, ou seja, com mais sintomas relatados pelos cuidadores do que o esperado para nesta faixa etária. Por sua vez, os escores estavam dentro da faixa de normalidade para problemas de condutas, hiperatividade, dificuldades com colegas e comportamento pró-social. No caso do CSHQ, ainda não há um ponto de corte estabelecido para a população brasileira. Mas, quando se compararam os dados obtidos nesta amostra com o critério de 41 pontos utilizado pelos estudos internacionais (Owens et al., 2000), observou-se uma média de 8 pontos acima do esperado, embora com um desvio padrão de 11 pontos, cujo limite inferior seria compatível com a pontuação de corte utilizada. Nesse sentido, estudos futuros com amostras representativas da população brasileira podem estabelecer um ponto de corte que seja mais compatível com as características de sono observadas no país.

Inicialmente, compararam-se os resultados entre meninos e meninas, com a finalidade de investigar possíveis diferenças nos escores que poderiam impactar a relação entre indicadores de saúde mental com problemas de sono. Os resultados obtidos mostraram ausência de diferenças estatisticamente significativas entre a pontuação de meninos e meninas tanto no escore total quanto nas subescalas do CSHQ e do SDQ, corroborando estudo prévio de Lianqi et al. (2004) realizado com crianças chinesas. As análises de correlação com CSHQ-BR foram então realizadas considerando a amostra como um todo.

Sabe-se que problemas de sono podem apresentar diferentes características (Nelson et al., 2018; Nunes \& Bruni, 2015), assim como há diferentes indicadores de problemas de saúde mental (Goodman, 1997). Por isso, optou-se por correlacionar as subescalas dos questionários para além dos seus escores totais.

No presente estudo, observou-se relação positiva entre pontuação total do CSHQ (indicativa de maiores dificuldades de sono) e problemas emocionais na infância, além de associação específica entre parassonias e distúrbios respiratórios do sono com as dificuldades emocionais. De acordo com os resultados, o aumento em características que indicam problemas de sono está associado ao aumento de indicadores de prejuízos na saúde mental relatados pelos familiares. Sabe-se que sintomas emocionais são cardinais em diversos transtornos de humor. Por sua vez, pesquisa recente discute dificuldades específicas do sono e relativamente comuns, como dificuldade para dormir sozinho e maior latência para início do sono, como preditores de depressão e gravidade de sintomas de ansiedade ao longo do tempo (Whalen et al., 2016). Alguns estudos relatam que, em crianças, sintomas emocionais e transtornos de 
sono podem estar relacionados com o tipo de maternagem, ou seja, quanto mais presente a mãe está na criação do seu filho, menos transtornos de sono e consequentemente menos problemas emocionais. As crianças pré-escolares que experimentam insegurança e ambivalência em seus relacionamentos de apego podem apresentar prejuízos na qualidade do sono (Schlarb et al., 2016). Nesse sentido, estudos futuros poderiam investigar se a relação entre problemas de sono e sintomas emocionais é mediada ou moderada por características de apego da criança em relação ao cuidador.

Observou-se que quanto mais tarde se inicia o sono, maior a pontuação na subescala de hiperatividade do SDQ, e que parassonias e distúrbios respiratórios do sono também se associam positivamente à hiperatividade. Isso indica que essas variáveis específicas dos aspectos do sono estão relacionadas a um aumento nos indicadores gerais de problemas de saúde mental. Esses resultados replicaram estudos anteriores, indicando uma associação universal entre distúrbios do sono e problemas comportamentais em crianças de diferentes países (Li et al., 2008; Nelson et al., 2018), especificamente com problemas externalizantes, como a hiperatividade (Wang et al., 2020). Esses autores encontraram correlações mais baixas entre parassonias, distúrbios respiratórios e início do sono com sintomas de hiperatividade quando comparadas às correlações observadas no presente estudo. Essas variações na magnitude das correlações podem ser decorrentes de número amostral, idade e aspectos culturais que influenciam tanto no sono quanto na saúde mental das crianças. Ressalta-se ainda o fato de que crianças na faixa etária de 7-8 anos com TDAH, ou seja, com prejuízos funcionais consistentes decorrentes dos sintomas de hiperatividade, dormem menos horas quando comparadas com as que não têm o distúrbio (Paavonen et al., 2009).

Em relação à idade, os achados do presente estudo também indicaram que quanto menor a idade da criança, mais horas de sono são relatadas pelos cuidadores. Notou-se que quanto maior a idade da criança, maior o tempo para início do sono, diminuindo, portanto, a quantidade de horas de sono da criança. Entre os fatores que podem justificar o início de sono tardio, nota-se o uso de dispositivos eletrônicos, como assistir à televisão antes de dormir, o que acarreta maior exposição à luz da tela, tornando assim a higiene do sono prejudicada. Uma hipótese seria a seguinte: com o aumento da idade, os cuidadores possuem menor controle sobre o uso noturno desses dispositivos. Sabe-se que o estilo de vida atual acarreta mudanças sociais que apresentam impacto negativo no padrão de sono, como despertares durante a noite, menor quantidade de horas de sono e, em alguns casos, insônia (Hoge et al., 2017). Houve também uma tendência para correlação entre idade e sonolência diurna, achado também observado por outros autores (Liu et al., 2019). Essa relação também pode ser explicada em função da quantidade menor de horas de sono conforme o aumento da idade. 
Em relação ao NSE, houve maior correlação com resistência em ir para a cama, indicando que quanto menor o NSE, maior a resistência. Discute-se a relação entre menor NSE e problemas relacionados ao sono, à medida que quartos compartilhados, estresse familiar, maior dificuldade no estabelecimento de limites e rotinas de sono poderiam implicar tais prejuízos (Crabtree et al., 2005; Li et al., 2008).

Por fim, no presente estudo, $23 \%$ das variâncias em indicadores internalizantes e externalizantes de saúde mental (escore total no SDQ) foram explicadas de forma significativa por características de sono, corroborando uma vasta literatura na área que relata alterações no padrão de sono como fator de risco e também critério diagnóstico em saúde mental (Nelson et al., 2018; Wang et al., 2020).

Este trabalho apresenta algumas limitações. A caracterização do sono foi medida a partir da versão abreviada do CSHQ-BR, sendo esta uma medida de relato dos cuidadores. A utilização combinada de medidas objetivas de sono, como a actigrafia e polissonografia, detaIharia melhor as características do sono nessa população. É importante que estudos adicionais avaliem o sono e o comportamento das crianças por meio de outros informantes, possibilitando melhor compreensão do funcionamento delas em diferentes contextos. A amostra deste estudo restringiu-se a 60 crianças de uma mesma escola pública de São Paulo. Espera-se que este trabalho possa estimular a produção de pesquisas nacionais sobre o tema, com amostras maiores e que contemplem a heterogeneidade populacional.

Todavia, uma importante contribuição deste estudo se deve ao fato de que os achados proveem as primeiras evidências de validade e precisão do CSHQ adaptado para o português do Brasil. O uso de ferramentas simples de rastreio em contexto escolar pode auxiliar na identificação de crianças de risco que devem ser encaminhadas para serviços de saúde mental, evitando a cronificação de sintomas que começaram na infância. Entender o padrão de sono como um possível fator de risco associado a transtornos psiquiátricos sinaliza a necessidade da investigação clínica pormenorizada sobre esse aspecto, de modo a facilitar o diagnóstico e a intervenção precoces, e modificar o prognóstico e curso de doença. 


\section{Referências}

Associação Brasileira de Empresas de Pesquisa (ABEP). (2019). Critério de Classificação Econômica Brasil. http://www.abep.org/criterio-brasil

Barbisan, B., \& Bueno, C. (2019). Sono normal na criança: A evolução do sono nas diversas idades. In B. Barbisan., C. Santos \& E. Motta. (Orgs.), Medicina do Sono (pp. 3-24). Atheneu.

Borsa, J. C., Damasio, B. F., \& Bandeira, D. R. (2012). Cross-cultural adaptation and validation of psychological instruments: Some considerations. Paidéia, 22(53), 423-432. https://doi. org/10.1590/S0103-863X2012000300014

Crabtree, V., Korhonen, J., Montgomery-Downs, H., Jones, V., O'Brien, L. \& Gozal, D. (2005). Cultural Influences on the bedtime behaviors of young children. Sleep Medicine, 6(4), 319-324. https:// doi.org/10.1016/j.sleep.2005.02.001

Cruz, L., Vizcaino, V. M., Bueno, C. A., Palencia, N. A., Lopez, M. S., Pacheco, B. N. (2016). Reability and validity of the Spanish version of the Children's Sleep Habits Questionnaire in school-age children. Child: Care, health and development, 42(6), 675-682. https://doi.org/10.1111/cch.12357

Damiani, J., Sweet, B., Sohoni, P. (2014). Melatonin: An option for managing sleep disorders in children with autism spectrum disorder. American Journal of Health-System Pharmacy, 71(2), 95-101. https://doi.org/10.2146/ajhp130215

Gios, T. S. (2020). Tradução e Adaptação Cultural para a Língua Portuguesa do Brasil do Questionário de Hábitos de Sono em Crianças (CSHQ-BR.) [Unpublished Master's Dissertation]. Faculdade de Ciências Médicas da Santa Casa de São Paulo.

Goodman, R. (1997). The Strengths and Difficulties Questionnaire: A research note. Journal of Child Psychology and Psychiatry, 38(5), 581-586. https://doi.org/10.1111/j.1469-7610.1997.tbo1545.x

Hoge, E., Bickham, D., \& Cantor, J. (2017). Digital media, anxiety, and depression in children. Pediatrics, 140(2), S76-S80. https://doi.org/10.1542/peds.2016-1758G

Li, S., Jin, X., Yan, C., Wu, S., Jiang, F., \& Shen, X. (2008). Bed- and room-sharing in Chinese school-aged children: Prevalence and association with sleep behaviors. Sleep medicine, 9(5), 555-563. https://doi.org/10.1016/j.sleep.2007.07.008

Lianqi, L., Chaojuan M., Ruzhan, W., Chunni, G., \& Xianchen, L. (2004). Sleep problems and related factors of elementary school students. Chinese Mental Health Journal, 18(9). https://en.cnki.com. cn/Article_en/CJFDTotal-ZXWS200409003.htm

Liu, X., Liu, L. \& Wang, R. (2003). Bed sharing sleep habits, and sleep problems among Chinese school-aged children. Sleep, 26(7), 839-844. https://doi.org/10.1093/sleep/26.7.839

Liu, Z., Wang, G., Tang, H., Wen, F., \& Li, N. (2014). Reliability and validity of the children's sleep habits questionnaire in preschool-aged Chinese children. Sleep and Biological Rhythms, 12(3), 187-193. https://doi.org/10.1111/sbr.2014.12.issue-3

Liu, Y., Zhang, J., Li, S. X., Chan, N. Y., Yu, M. W. M., Lam, S. P., \& Wing, Y. K. (2019). Excessive daytime sleepiness among children and adolescents: Prevalence, correlates, and pubertal effects. Sleep Medicine, 53, 1-8. https://doi.org/10.1016/j.sleep.2018.08.028 
Loekmanwidjaja, J., Carneiro, A. C. F., Nishinaka, M. L. T., Munhoes, D. A., Benezoli, G., Wandalsen, G. F., \& Solé, D. (2018). Distúrbios do sono em crianças com rinite alérgica persistente moderada-grave. Brazilian Journal of Otorhinolaryngology, 84(2), 178-184. https://doi.org/10.1016/j. bjorl.2017.01.008

Miano, S., Parisi, P., \& Villa, M. P. (2012). The sleep phenotypes of attention deficit hyperactivity disorder: The role of arousal during sleep and implications for treatment. Med Hypotheses, 79(2), 147-153. https://doi.org/10.1016/j.mehy.2012.04.020

Moore, M., Evans, V., Hanvey, G., Johnson, C. (2017). Assesment of sleep in children with autism spectrum disorder. Children, 4(8), 72. https://doi.org/10.3390/children4080072

Nelson, T. D., Kidwell, K. M., Hankey, M., Nelson, J. M., \& Espy, K. A. (2018). Preschool executive control and sleep problems in early adolescence. Behavioral Sleep Medicine, 16(5), 494-503. https://doi.org/10.1080/15402002.2016.1228650

Neves G., Macedo P., \& Gomes M. (2017). Transtornos do Sono: Atualização. Revista Brasileira de Neurologia, 53(3), 19-30. http://docs.bvsalud.org/biblioref/2017/12/876873/rbn-533-3-transtornos-do-sono-1-2.pdf

Nunes, M. L., \& Bruni, O. (2015). Insomnia in childhood and adolescence: Clinical aspects, diagnosis and therapeutic approach. Jornal de Pediatria, 91(6), 26-35. https://doi.org/10.1016/j. jped.2015.08.006

Owens, J. A., Spirito, A., \& McGuinn, M. (2000). The Children's Sleep Habits Questionnaire (CSHQ): Psychometric properties of a survey instrument for school-aged children. Sleep, 15;23(8), 1043-1051. https://doi.org/10.1037/t33022-000

Owens, J. A., \& Mindell, J. A. (2011). Pediatric Insomnia. Pediatr Clin N Am, 58, 555-569. https://doi. org/10.1016/j.pcl.2011.03.011

Paavonen, E. J., Räikkönen, K., Lahti, J., Komsi, N., Heinonen, K., Pesonen, A. K., Jarvenpaa, A., Strandberg, T., Kajantie, E., \& Porkka-Heiskanen, T. (2009). Short sleep duration and behavioral symptoms of attention-deficit/hyperactivity disorder in healthy 7-to 8-year-old children. Pediatrics, 123(5), e857-e864. https://doi.org/10.1542/peds.2008-2164

Saur, A. M., \& Loureiro, S. R. (2012). Qualidades psicométricas do Questionário de Capacidades e Dificuldades: Revisão da literatura. Estudos de Psicologia (Campinas), 29(4), 619-629.

Schlarb, A. A., Jaeger, S., Schneider, S., In-Albon, T., \& Hautzinger, M. (2016). Sleep problems and separation anxiety in preschool-aged children: A path analysis. Journal of Child and Family Studies, 25(3), 902-910. https://doi.org/10.1007/s10826-015-0262-z

Schlarb, A.A., Schwerdtle, B., Hautzinger, M. (2010). Validation and psychometric properties of the German version of the Children's Sleep Habits Questionnaire. Somnologie. Schiafforschung und Schlafmedizin, 14, 260-266. https://doi.org/10.1007/s11818-010-0495-4

Silva, T. B., Osório, F. L., \& Loureiro, S. R. (2015). SDQ: Discriminative validity and diagnostic potential. Frontiers in Psychology, 6, 811. https://doi.org/10.3389/fpsyg.2015.00811

Silva, F. G., Silva, C. R., Braga, L. B., \& Neto, A. S. (2014). Portuguese Children's Sleep Habits Questionnaire- validation and cross-cultural comparison. Jornal de Pediatria, 9o(1), 78-84. http:// dx.doi.org/10.1016/j.jped.2013.06.009 
Tso, W., Rao, N., Jiang, F., Li, A. M., Lee, S. L., Li, S., Ip, P., \& Ho, F. K. (2016). Sleep duration and school readiness of Chinese preschool children. The Journal of Pediatrics, 169(11), 266-271. https://doi. org/10.1016/j.jpeds.2015.10.064

Tzchishinsky, O., Lufi, D., Shochat, T. (2008). Reliability of the Children's Sleep Habits Questionnaire Hebrew Translation and Cross Cultural Comparison of the Psychometric Properties. Sleep Diagnosis and Therapy, 3(3). https://sleepdt.com/reliability-of-the-childrens-sleep-habitsquestionnaire-hebrew-translation-and-cross-cultural-comparison-of-the-psychometric-properties/

Urrutia-Pereira, M., Solé, D., Neto, H. C., Acosta, V., Cepeda, A. M., Álvarez-Castelló, M., Almendarez, J., Lozano-Saenz, J. C., Sisul-Alvariza, N. A., Rosario, A. J., Castillo, M., Valentin-Rostan, H., Badellino, R. L., Castro-Almarales, M., González-León, C., Sanchez-Silot, M. M., Avalos, C., Fernandez, F., Berroa, M. M., De la Cruz, R. O. S. \& Sarni, R. S. (2017). Sleep disorders in Latin-American children with asthma and/or allergic rhinitis and normal controls. Allergologia et immunopathologia, 45(2), 145-151. https://doi.org/10.1016/j.aller.2016.05.005

Vaughn, B. E., Elmorestaton, L., Shin, N., \& Elsheikh, M. (2015). Sleep as a support for social competence, peer relations, and cognitive functioning in preschool children. Behavioral Sleep Medicine, 13(2), 92-106. doi:10.1080/15402002.2013.845778.

Waumans, R. C., Terwee, C. B., Van den Berg, G., Knol, D. L., Van Litsenburg, R. R. L., Gemke, R. J. (2010). Sleep and Sleep Disturbance in Children: Reliability and Validity of the Dutch Version of the Child Sleep Habits Questionnaire. Sleep, 33(6), 841-845. https://doi.org/10.1093/ sleep/33.6.841

Whalen, D. J., Gilbert, K. E., Barch, D. M., Luby, J. L., \& Belden, A. C. (2016). Variation in common preschool sleep problems as an early predictor for depression and anxiety symptom severity across time. Journal of Child Psychology \& Psychiatry, 58(2), 151-159. https://doi.org/10.1111/ jcpp.12639

Wang, G., Takahashi, M., Wu, R., Liu, Z., Adachi, M., Saito, M., Nakamura, K., \& Jiang, F. (2020). Association between Sleep Disturbances and Emotional/Behavioral Problems in Chinese and Japanese Preschoolers. Behavioral Sleep Medicine, 18(3), 420-431. https://doi.org/10.1080/15402 002.2019 .1605995

Wu, X., Tao, S., Rutayisire, E., Chen, Y., Huang, K., \& Tao, F. (2016). The relationship between screen time, nighttime sleep duration, and behavioural problems in preschool children in China. EuropeanChild \&Adolescent Psychiatry, 26(5), 541-548. https://doi.org/10.1007/s00787-016-0912-8

Xie, Z., Chen, F., Li, W., Geng, X., Li, C., Meng, X., Feng, Y., Liu, W., \& Yu, F. (2017). A review of sleep disorders and melatonin. Neurological Research, 39(6), 559-565. https://doi.org/10.1080/01616 412.2017.1315864 
EQUIPE EDITORIAL

\section{Editora-chefe}

Cristiane Silvestre de Paula

\section{Editores associados}

Alessandra Gotuzo Seabra

Ana Alexandra Caldas Osório

Luiz Renato Rodrigues Carreiro

Maria Cristina Triguero

Veloz Teixeira

Editores de seção

"Avaliação Psicológica"

Alexandre Serpa

André Luiz de Carvalho

Braule Pinto

Luiz Renato Rodrigues Carreiro

Vera Lúcia Esteves Mateus

\section{"Psicologia e Educação"}

Alessandra Gotuzo Seabra

Carlo Schmidt

Regina Basso Zanon
"Psicologia Social e Saúde das

Populações"

Enzo Banti Bissoli

Marina Xavier Carpena

"Psicologia Clínica"

Carolina Andrea Ziebold Jorquera

Julia Garcia Durand

Natalia Becker

"Desenvolvimento Humano"

Maria Cristina Triguero

Veloz Teixeira

Rosane Lowenthal

\section{Suporte técnico}

Maria Fernanda Liuti

Bento da Silva

Camila Fragoso Ribeiro
PRODUÇÃO EDITORIAL

Coordenação editorial

Ana Claudia de Mauro

Estagiários editoriais

Júlia Lins Reis

Preparação de originais

Carlos Villarruel

\section{Revisão}

Vera Ayres

Diagramação

Acqua Estúdio Gráfico 


\title{
Appendix 1. CSHQ-BR (abbreviated version)
}

\author{
Questionário de Hábitos de Sono em Crianças (CSHQ-BR) \\ (versão abreviada/ pré-escolar e escolar)
}

Pontuação total

Seguem abaixo afirmações sobre hábitos e possíveis dificuldades de sono das crianças. Pense na última semana do seu filho(a) para responder às questões. Se a última semana foi atípica por algum motivo específico, escolha uma semana comum mais recente. Responda USUALMENTE se ocorreu 5 ou mais vezes na semana; responda ÀS VEZES se ocorreu entre 2 e 4 vezes na semana; responda RARAMENTE se ocorreu uma vez na semana ou nunca.

Observação: substituir a palavra "cama" por "rede", se necessário.

\section{Hora de dormir}

Escreva a hora que a criança vai dormir:

3

2

1

USUALMENTE ÀS VEZES RARAMENTE

$(5-7) \quad(2-4) \quad(0-1)$

1) A criança vai para a cama sempre na mesma hora

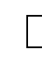

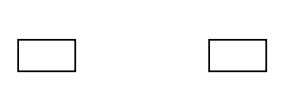

2) A criança adormece dentro de 20 minutos após

ir para a cama
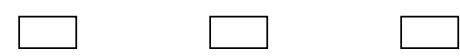

3) A criança adormece sozinha na sua própria cama
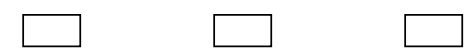

4) A criança adormece na cama dos pais ou dos irmãos

5) A criança precisa da presença dos pais no quarto para adormecer

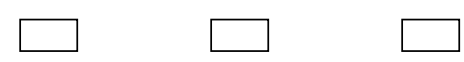


6) A criança luta contra o sono na hora de dormir (chora, se recusa a ficar na cama...)

7) A criança tem medo de dormir no escuro

8) A criança tem medo de dormir sozinha

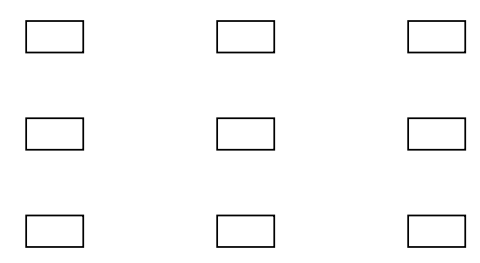

\section{Hábitos de sono}

Quantidade habitual de sono por dia: horas e minutos

(levar em conta as horas de sono da noite e sonecas)

9) A criança dorme pouco

10) A criança dorme a quantidade certa

11) A criança dorme o mesmo tempo de sono todos os dias

12) A criança molha a cama à noite
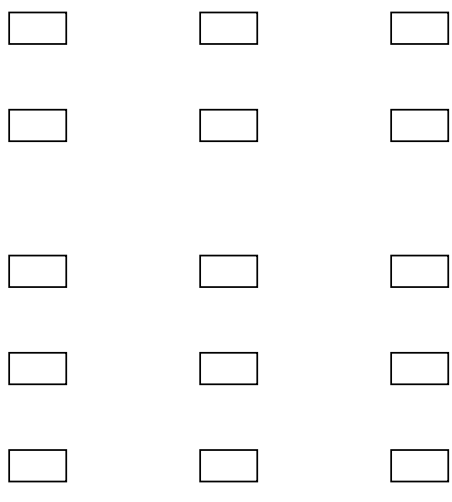

13) A criança fala durante o sono

14) A criança fica inquieta e se move bastante durante o sono
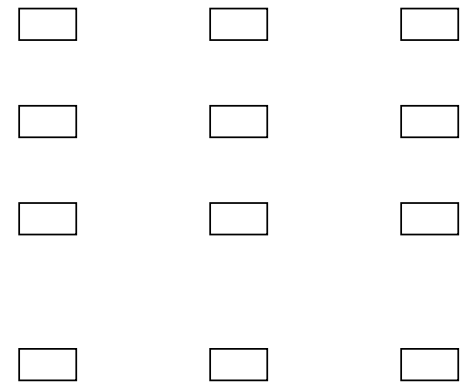

15) A criança caminha enquanto dorme

16) A criança vai para a cama de outra pessoa durante a noite (pais, irmão, irmã...) 
17) A criança range os dentes durante o sono (seu dentista pode ter mencionado isso)

18) A criança ronca alto

19) A criança parece parar de respirar durante o sono

20) A criança bufa e/ou suspira durante o sono

21) A criança tem dificuldade para dormir longe de casa (quando visita parentes, férias...)

22) A criança acorda durante a noite gritando, suando e inconsolável

23) A criança acorda agitada após pesadelo

\section{Acordar durante a noite}

3

USUALMENTE ÀS VEZES RARAMENTE

$(5-7) \quad(2-4) \quad(0-1)$

24) A criança acorda uma vez durante a noite
2

1
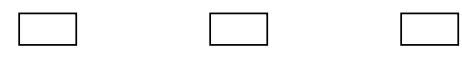
25) A criança acorda mais de uma vez durante
a noite

Escreva quantos minutos duram os despertares noturnos: 


\section{Acordar pela manhã}

Escreva a hora que a criança acorda pela manhã:

\section{3}

2

1

\section{USUALMENTE ÀS VEZES RARAMENTE}

$(5-7) \quad(2-4) \quad(0-1)$

26) A criança acorda sozinha
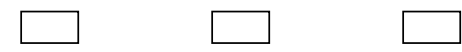

27) A criança acorda mal-humorada

28) A criança é acordada por um adulto ou irmão
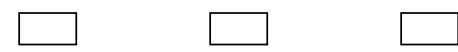

29) A criança tem dificuldade em levantar da cama pela manhã
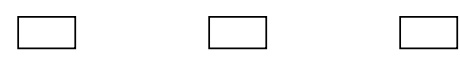

30) A criança leva bastante tempo para ficar alerta pela manhã
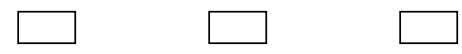

\section{Sonolência durante o dia}

31) A criança parece cansada

A criança apresentou muito sono, adormeceu ou ficou sem sono durante as seguintes atividades:
1
2
3
Não

Sem sono Muito sono Adormeceu Aplicável

32) Assistindo TV

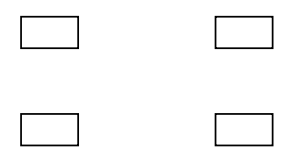

33) Andando de carro 